\title{
Development of a Comparative Risk Ranking System for Agents Posing a Bioterrorism Threat to Human or Animal Populations
}

Katharina Tomuzia, Andrea Menrath, Hendrik Frentzel, Matthias Filter, Armin A. Weiser, Juliane Bräunig, Anja Buschulte, and Bernd Appel

Various systems for prioritizing biological agents with respect to their applicability as biological weapons are available, ranging from qualitative to (semi)quantitative approaches. This research aimed at generating a generic risk ranking system applicable to human and animal pathogenic agents based on scientific information. Criteria were evaluated and clustered to create a criteria list. Considering availability of data, a number of 28 criteria separated by content were identified that can be classified in 11 thematic areas or categories. Relevant categories contributing to probability were historical aspects, accessibility, production efforts, and possible paths for dispersion. Categories associated with impact are dealing with containment measures, availability of diagnostics, preventive and treatment measures in human and animal populations, impact on society, human and veterinary public health, and economic and ecological consequences. To allow data-based scoring, each criterion was described by at least 1 measure that allows the assignment of values. These values constitute quantities, ranges, or facts that are as explicit and precise as possible. The consideration of minimum and maximum values that can occur due to natural variations and that are often described in the literature led to the development of minimum and maximum criteria and consequently category scores. Missing or incomplete data, and uncertainty resulting therefrom, were integrated into the scheme via a cautious (but not overcautious) approach. The visualization technique that was used allows the description and illustration of uncertainty on the level of probability and impact. The developed risk ranking system was evaluated by assessing the risk originating from the bioterrorism threat of the animal pathogen bluetongue virus, the human pathogen Enterohemorrhagic Escherichia coli O157:H7, the zoonotic Bacillus anthracis, and Botulinum neurotoxin.

$\mathrm{T}$ HE INTENTIONAL DissEMination OF Biological AGENTS to cause illness or death in people as well as direct or indirect economic losses due to the disruption of the food supply or damage to the public or animal health sector poses a great challenge for decision makers. There- fore, an objective and comprehensive risk ranking system is necessary for the description and evaluation of risk emanating from different biological agents. ${ }^{1}$

Several biological agents (bacteria, viruses, and toxins) can cause severe diseases and are seen as a potential threat

Dr. Katharina Tomuzia, Dr. Andrea Menrath, Hendrik Frentzel, Matthias Filter, Dr. Armin A. Weiser, and Dr. Anja Buschulte are Research Scientists; Dr. Juliane Bräunig is Head of Unit Microbial Toxins; and Prof. Bernd Appel is Head of the department; all in the Department Biological Safety, Federal Institute for Risk Assessment (BfR), Berlin, Germany. 
with regard to bioterrorism. Besides well-known agents like Bacillus anthracis or Botulinum neurotoxin, viral hemorrhagic fever viruses and newly emerging agents like the yellow fever virus are also listed in various prioritizing lists. $^{2-5}$

In order to set guidelines for decision makers and stakeholders, it is necessary to conduct a risk assessment of specific biological agents. One way of doing so is to create a risk ranking that categorizes the agents according to their feasibility to be used as threat agents as well as their impacts in case of use in a bioterrorism incident. ${ }^{6}$

A ranking system can be created by various methodseither a qualitative or a (semi)quantitative approach can be used - and the exact definition of risk must be determined. Fosse et al. established a quantitative approach based on the creation of a typology of hazards and calculated a risk score to support veterinary public health decision makers. ${ }^{7}$ This very precise system is generated out of detailed data for each ranked agent.

The crucial point for conducting a reliable quantitative risk ranking is the quality of underlying data. For exact quantitative risk rankings, the data necessarily have to be quantitative and precise. Unfortunately, these exact data are not always available for emerging or rare pathogens. Additionally, the collection of quantitative data is usually only feasible in crisis preparation, whereas time limitations in emergency situations would not allow the collection of information with the necessary exactness.

In contrast, Davis ${ }^{8}$ as well as Franz et al. ${ }^{9}$ and Irlenkäuser ${ }^{10}$ developed qualitative risk ranking systems that allow differentiation between hazardous agents and probably nonthreatening agents in an approximate manner and without gradation between risk originating from the different agents. However, for optimal preparedness and containment, it would be useful to separate the ranked agents according to their posed threat and relevance. A qualitative risk ranking, in contrast to a quantitative risk ranking, is often based on expert opinions or estimated data, and therefore it can be conducted even if there is a medium to low level of knowledge about an agent. ${ }^{5,11-15}$ A middle approach is risk evaluation in a semiquantitative manner. This is a compromise regarding the preciseness of the results, but the complexity and duration of this kind of assessment as well as its dependence on exact data is lower than for quantitative risk rankings. ${ }^{2,13,15-18}$

A detailed overview on available risk assessment methods, their focus, and risk ranking structures is presented elsewhere. ${ }^{19}$ It is most crucial to develop a risk ranking scheme for highly pathogenic agents that is generic and therefore usable for all fields of application and all target populations. ${ }^{19}$ This would ideally lead to a universally accepted risk ranking system for the support of decision makers and stakeholders in emergency situations.

\section{Methods}

Based on the literature survey already published, we revised the criteria and compared them in order to get an overview of the most important factors various authors identified for their risk ranking systems. ${ }^{19}$ Since most criteria describe only abstract concepts, like accessibility, it was additionally necessary to determine measures (desirably measurable parameters) that characterize each criterion explicitly. The measures were chosen carefully so that they would be unambiguous within the set of criteria; it also was preferable that they represent parameters that can be characterized with explicit information like a number, defined range, or clear definitions and facts. Special care was taken that criteria were formulated that describe unique concepts and that could be assigned unambiguously to categories. Criteria that were originally used to describe different categories in other ranking systems were evaluated according to their main contributing aspect and assigned to the respective category. The same approach was used on the measure level. Each measure was assigned to 1 criterion so that each criterion was described by at least 1 measure. Relevant criteria were clustered in 11 categories representing miscellaneous perspectives with regard to a bioterrorism incident, starting with the agent selection and ending with the impact on public health and other consequences caused by the release of the agent (see Figure 1).

As a next step, a literature search was performed regarding the range of data of measures and agents to assess which measures can be used to describe high-risk biological agents comprehensively and objectively. According to our literature survey, it was assessed whether data for the measures could be obtained in general or for the examined highly pathogenic agents in particular. For example, the measure "stability of the agent in the environment" was deleted from the criteria set because no complete global data coverage exists that would presume to make a point regarding this complex measure. Furthermore, it has to be considered that, for example, the measure "stability of the agent in the environment" cannot be unambiguously assigned to 1 criterion or category. It can be used to describe the prevalence of the agent in the environment and therefore the accessibility of a specific agent to a perpetrator. Additionally, stability can be used to describe the shelf-life of a biological agent and thus the production efforts and storability. Moreover, stability has implications for the agent's dispersal in various matrices and hence influences directly human and veterinary health. In order to avoid overlap in the criterion "accessibility in general," the measure "stability" was not used additionally, since it was already included in the measure "prevalence in the environment." Also, some measures were deleted because appropriate data provision turned out not to be feasible.

In summary, the assignment of the measures was used to describe the criteria as exactly as possible. So the criteria 


\section{Categories}

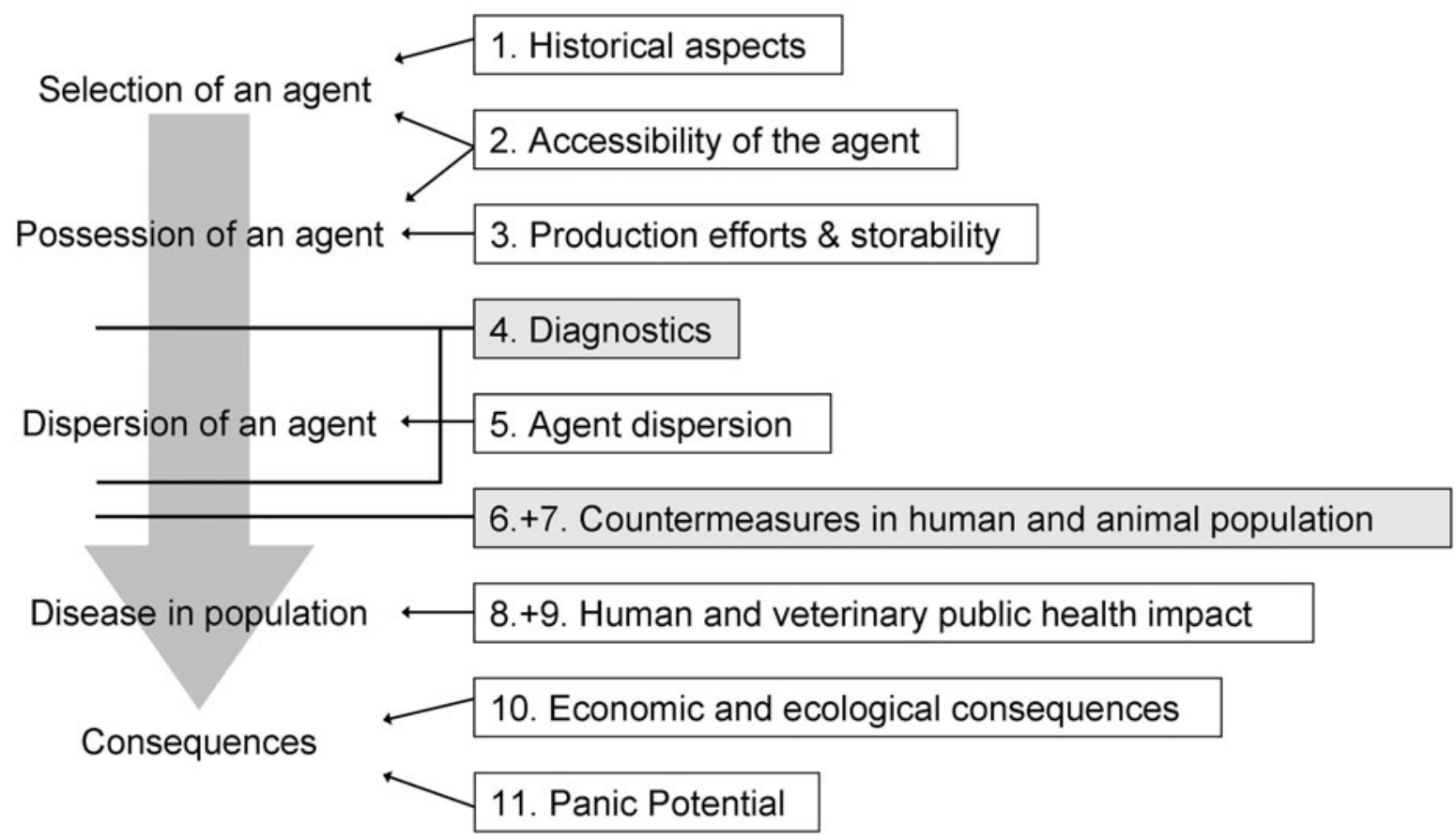

Figure 1. Flow chart of categories used for ranking highly pathogenic biological agents to assess the risk related to a bioterrorism scenario (right-hand side: list of the 11 categories, white boxes contain fact and analytical categories; grey boxes with black barriers include categories with criteria and measures for the containment of the agent)

cannot be equated with data regarding the biological agents because the defined criteria often are described by a combination of several measures to include different aspects. This constitutes the main difference with other risk ranking systems of highly pathogenic biological agents where criteria are used directly for the characterization of the agents. $^{13,14}$

As a result of this approach, we created a hierarchical evaluation schema that is grounded on 51 measures describing 28 criteria, which in turn are unambiguously assigned to 11 descriptive categories that encode the risk perspectives "probability" and "impact" (Table 1).

In order to allow a comparative ranking of different high-risk biological agents, each measure was graded in a class ranging from " 1 " to " 4 " following the method of McKenzie et al. ${ }^{20}$ The gradation into 4 classes allows a clear and easily distinguishable subdivision of values for each measure and avoids center values for measures where expert opinion is needed. It is well known from surveys that the center value is predominantly chosen for measures where no explicit quantitative data are available but a qualitative estimation is at choice. It has to be kept in mind that the value of " 1 " refers to the classification of "none to low." This implies that a disease with no morbidity is scored in the lowest possible class (1), which includes as well, for example, diseases that have low lethality.

Depending on the source employed and the information available, agent data were classified into 1 of the 4 classes according to the adopted gradation. If epidemiologic data were available, the median was calculated to eliminate discordant values - for example, for the number of cases, morbidity rate or case-fatality rate. For this purpose, the median (eg, of numbers of cases) of the past 5 years was calculated to depict the most comprehensive picture of the disease. If no epidemiologic data were available, arithmetic means of mentioned values out of several references were used to characterize the specific biological agent. Additionally, the minimum and maximum values of the measure values formed the basis for generating possible minimum and maximum classes to illustrate the bandwidth of the real data including natural variations. This concept was also used in other risk ranking systems. ${ }^{5}$

Then, the final value of each criterion was calculated as the arithmetic mean value of all measures associated with the respective criterion. Subsequently, the arithmetic mean values of all criteria values were used to compose a mean value for their assigned category. These mean category values are in turn used to calculate the 2 multiplying perspectives of risk: probability and impact as arithmetic means of the category values (Figure 2).

The same calculations were also conducted for the minimum and maximum values on each level to allow for the visualization of uncertainty. Risk is defined as the product of probability and impact by the ISO definition as well as by Salerno et al. and Tucker ${ }^{21-23}$ and is therefore calculated this way in the ranking schema as well. This 


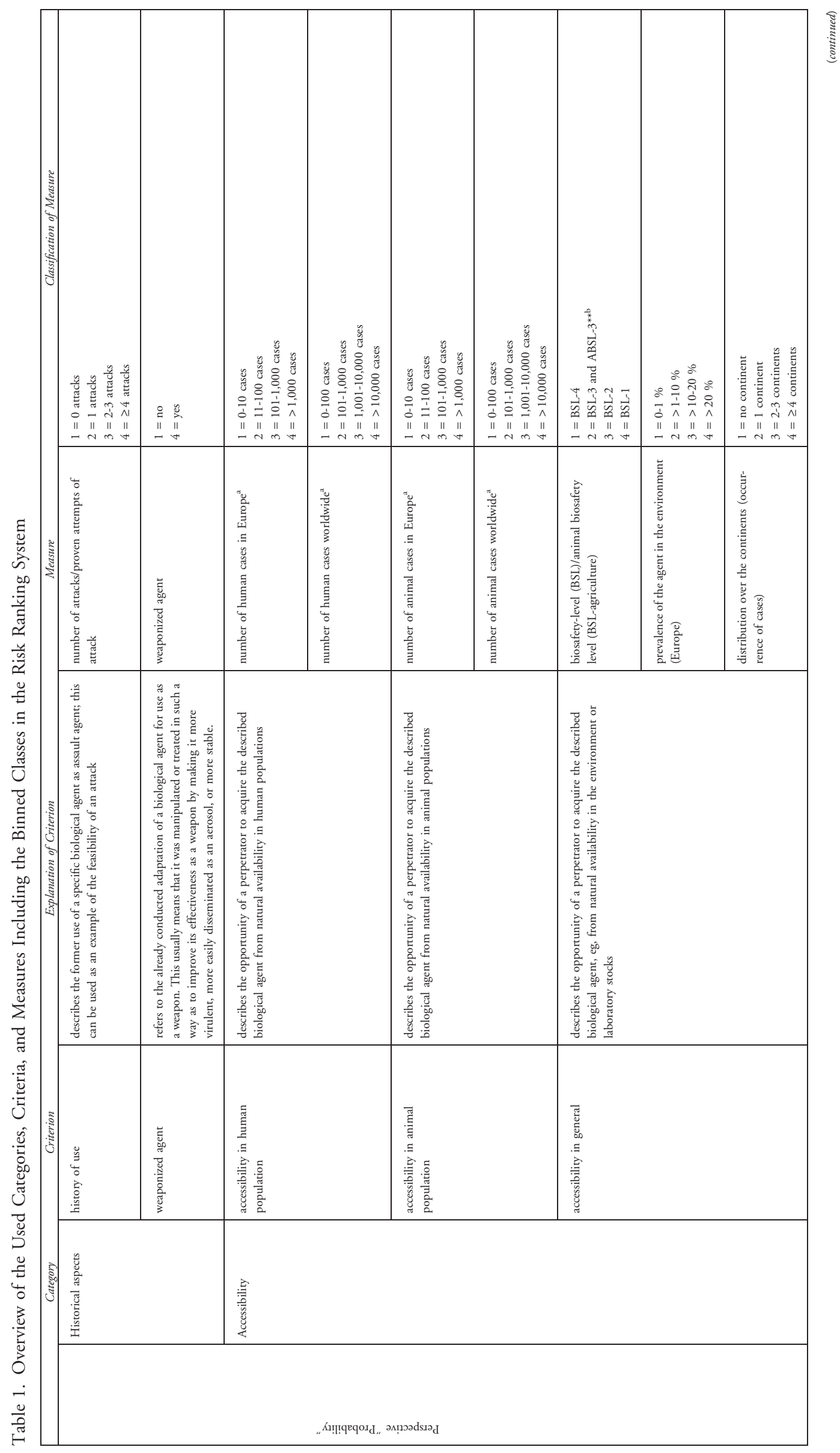




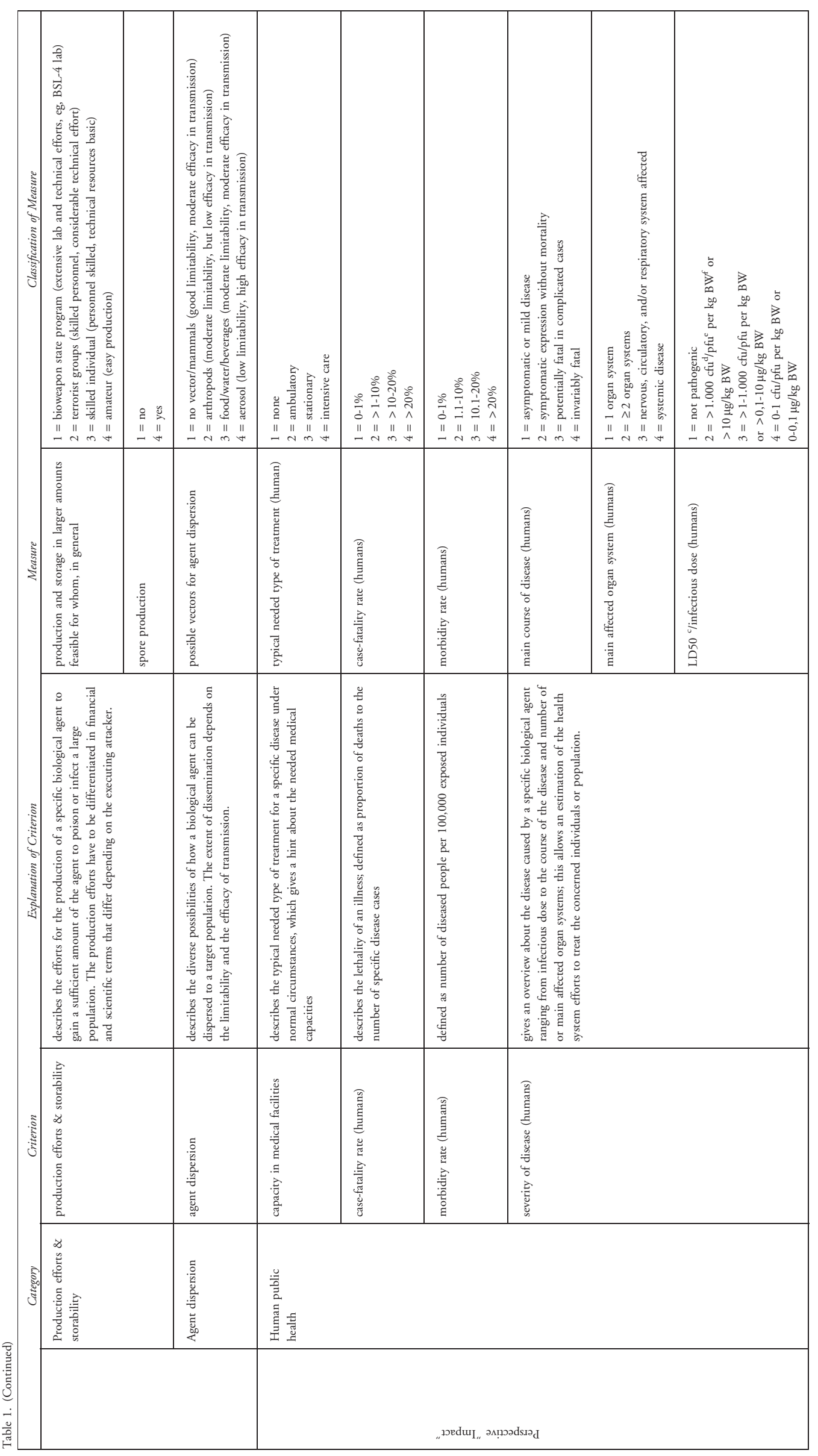




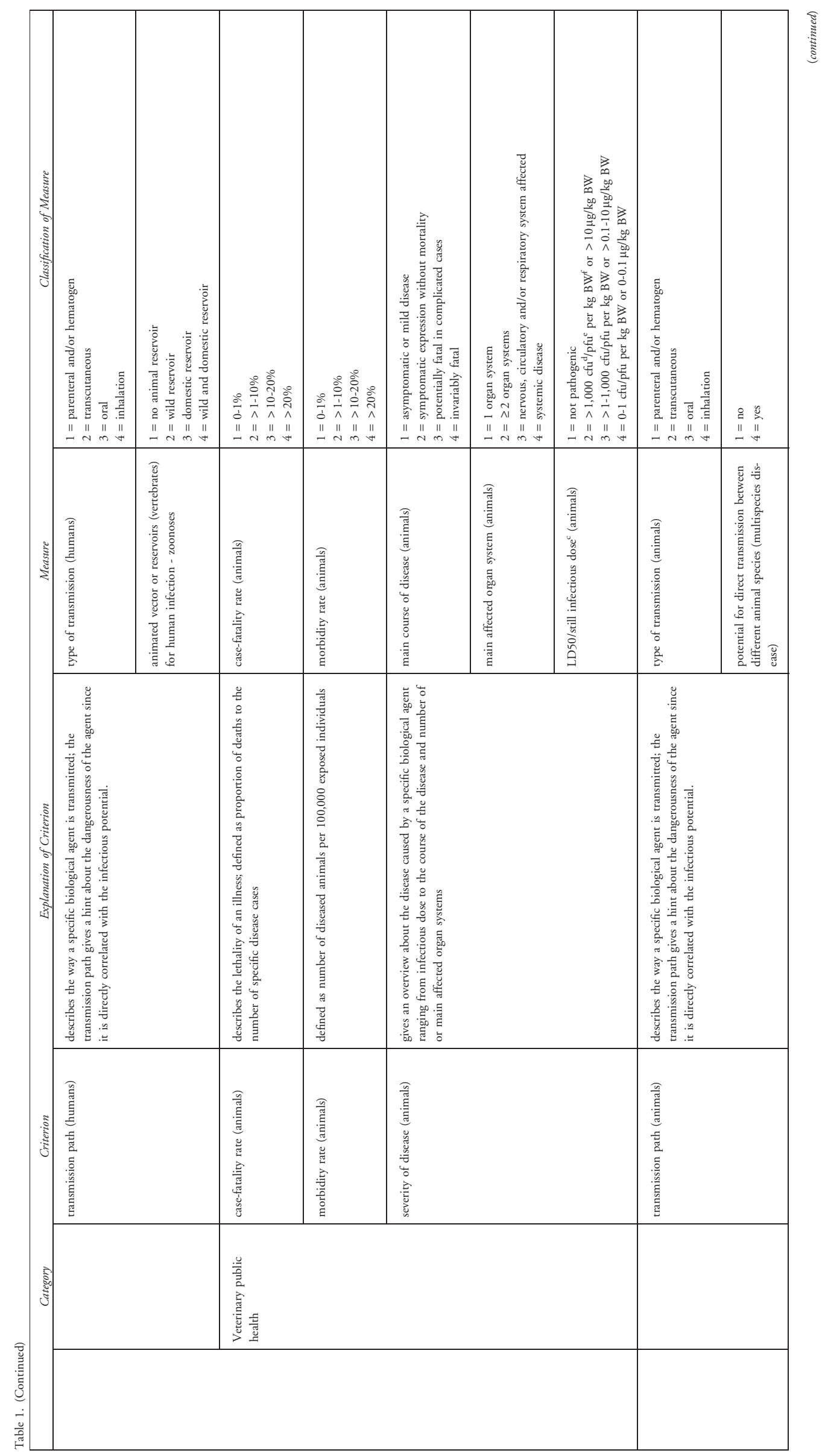




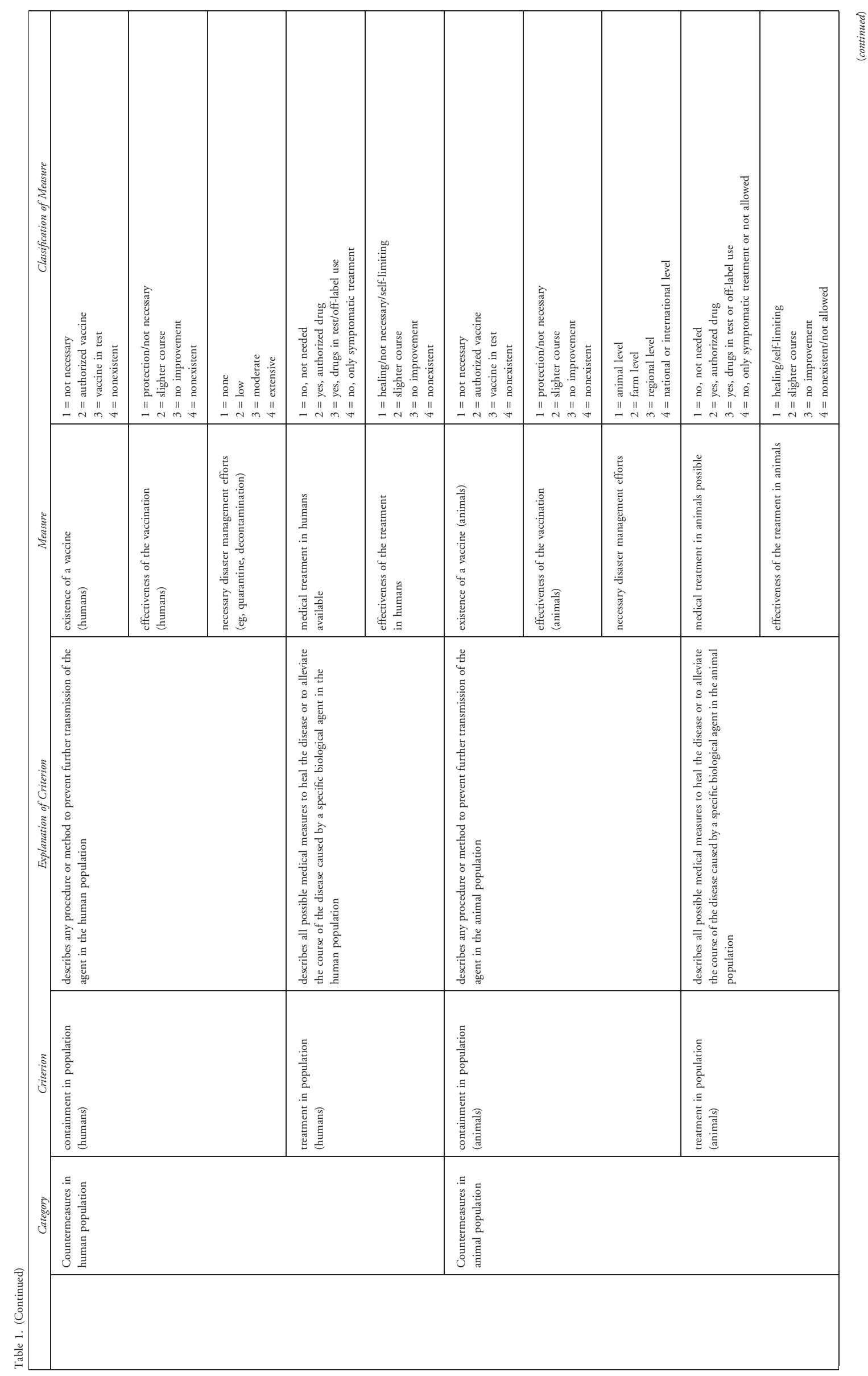

Volume 11, Supplement 1, 2013 


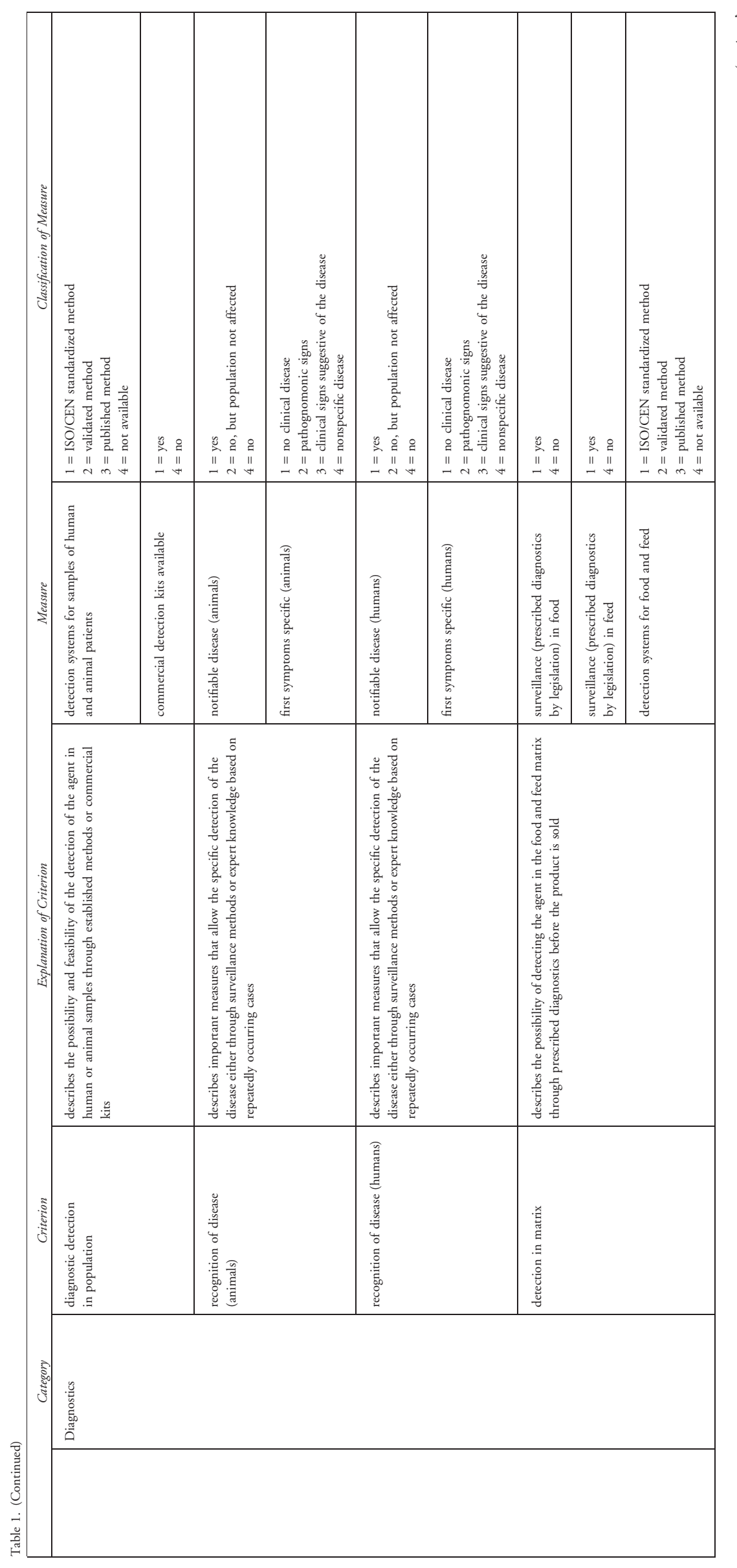




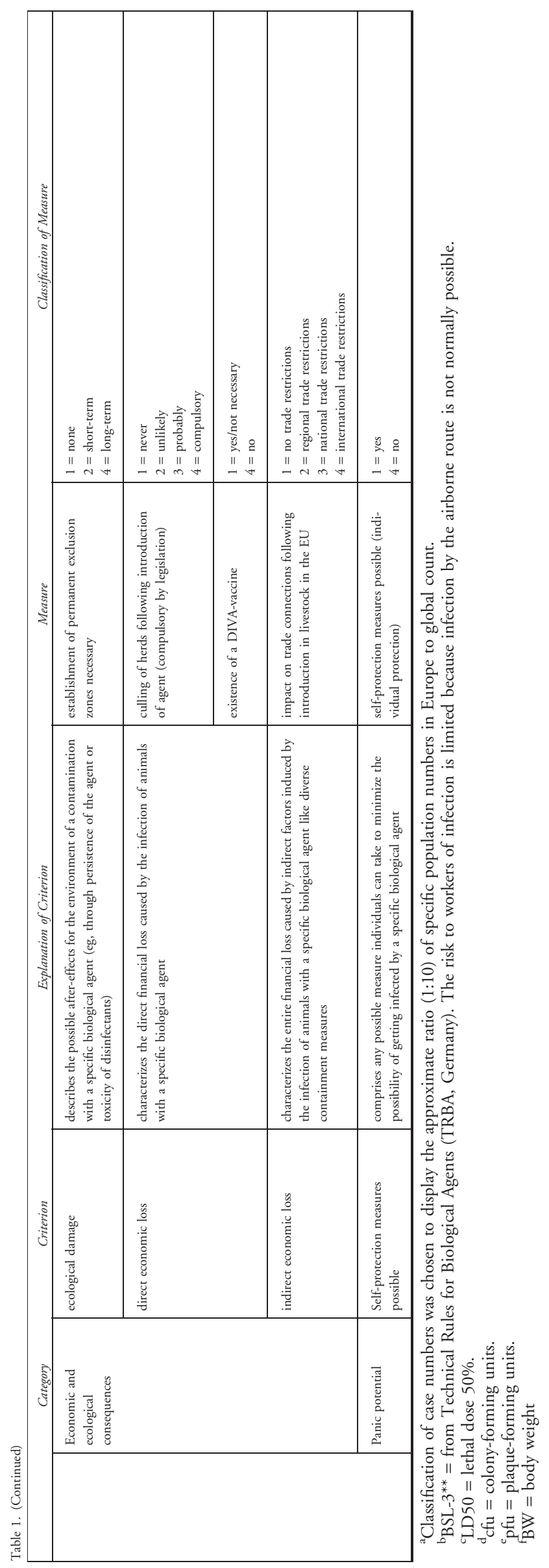




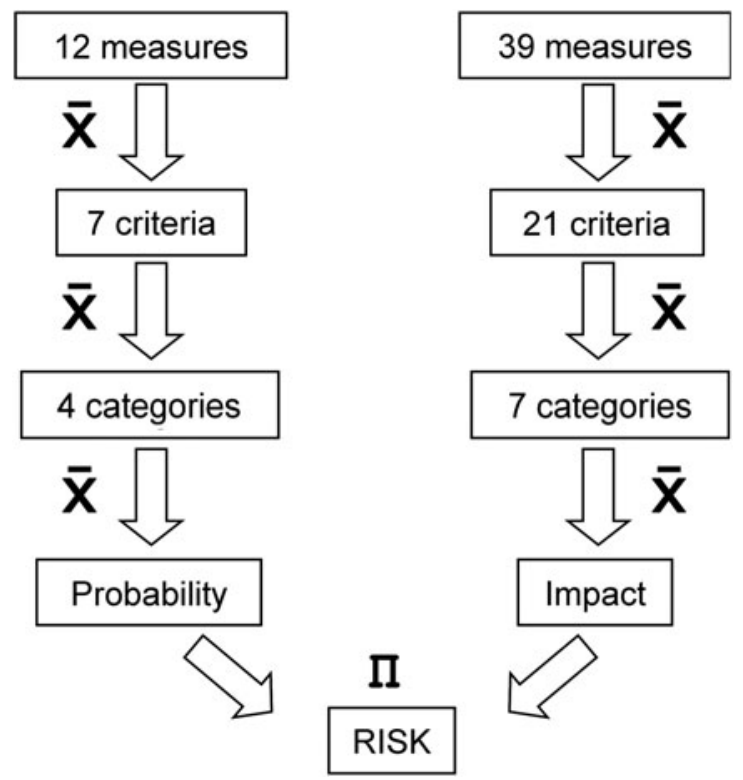

Figure 2. Description of the calculation of risk from measures, criteria, and categories to probability or impact and risk. Values for each criteria, category, and probability and impact originate from the arithmetic mean of the level before. In contrast, risk is defined as the product of probability and impact. ${ }^{21}$

enables the visualization of risk in a probability-impact matrix according to Salerno et al. ${ }^{22}$

All calculations and visualization were performed using a VBA (Visual Basic for Applications) program in combination with a Microsoft Excel $^{\circledR}$ database that provides the necessary data. Inserted raw data are automatically categorized in the presented classes, calculation steps as described above are conducted, and final values are determined and visualized. The presented risk ranking system additionally provides the possibility for weighting on each of the hierarchical levels (measures, criteria, categories). These weighting factors could be applied by skilled users to focus the risk ranking on specified populations or to adapt the system to individual demands. For a basic risk ranking, as presented in this article, the values for the biological agents were calculated using equal weighting factors of 1 on each level.

\section{Results}

The grading system of the developed risk ranking is presented in Table 1. The table describes which categories determine the risk perspectives probability and impact. The chosen categories for the description of probability were the history of use, the accessibility of the agent, and possible paths of introduction and contamination as well as the feasibility of agent production and storage. For the complete estimation of the impact, it is necessary to consider not only the agent's effects on human and veterinary public health, but also possible countermeasures that could contain the spread of the disease (vaccination and treatment) within the possible target populations. In addition, the diagnostic aspects as well as the economic and socioeconomic consequences and potential for panic represent issues that influence the impact caused by a specific biological agent. Moreover, Table 1 illustrates the assignment of criteria to the specific category for a more precise description of the aspect as well as an explanation and the describing measurable parameters (measures) for each criterion. Furthermore, the underlying classification of the measures with its class limits is given.

Some measures that are used in other publications for the description of a specific criterion or category might be arranged in a different manner in the developed ranking system. For example, "number of human/animal cases in Europe/world" is not used to describe how hazardous an agent is because of its naturally occurring prevalence but to depict the perspective of accessibility to the specific biological agent for a potential perpetrator. ${ }^{5,13,14,24}$

By using the minimum and maximum values of the measure values, the basis for the generation of possible minimum and maximum classes was provided to illustrate the data variation caused by differences in data availability or natural variability, for example. It is possible that some measures like "case-fatality rate" cover broad ranges, with the result that the variation of data for this measure would include 2 or even 3 classes. In order to solve this problem, a mean or if possible a median was averaged over the range of epidemiologic data-for example, a value of $22.5 \%$ as the arithmetic mean if the bandwidth was $5 \%$ to $40 \%$. The variation was delineated through the mentioned minimum and maximum measure classes (on the basis of the chosen example with a minimum of $5 \%$ and a maximum of $40 \%$ ). These minimum and maximum measure classes were thus considered when calculating the value of the criteria. Like the minimum and maximum criteria classes, minimum and maximum category values also were generated and could be shown visually as data uncertainty boxes in the resulting probability-by-impact matrix (presented in Figure 3 on the basis of 4 biological agents).

This means that we neither ignore uncertainty by assigning no value nor delete the specific criterion or measure. Instead, we outline and represent the uncertainty in a clearly structured and comprehensible manner. The uncertainty boxes around the scores represent minimum and maximum values of available data. Uncertainty is particularly great for many exotic or newly emerging viruses. Risk analysts can assess what the risk of a specific biological agent might be by examining the uncertainty boxes. Every value within the area of the uncertainty boxes is realistic and could occur, but statistically the median or mean value is the most probable value and therefore used as a central value in the risk ranking (Figure 3).

Another challenge that emerged during the data collection for high-risk biological agents constitutes the entire 


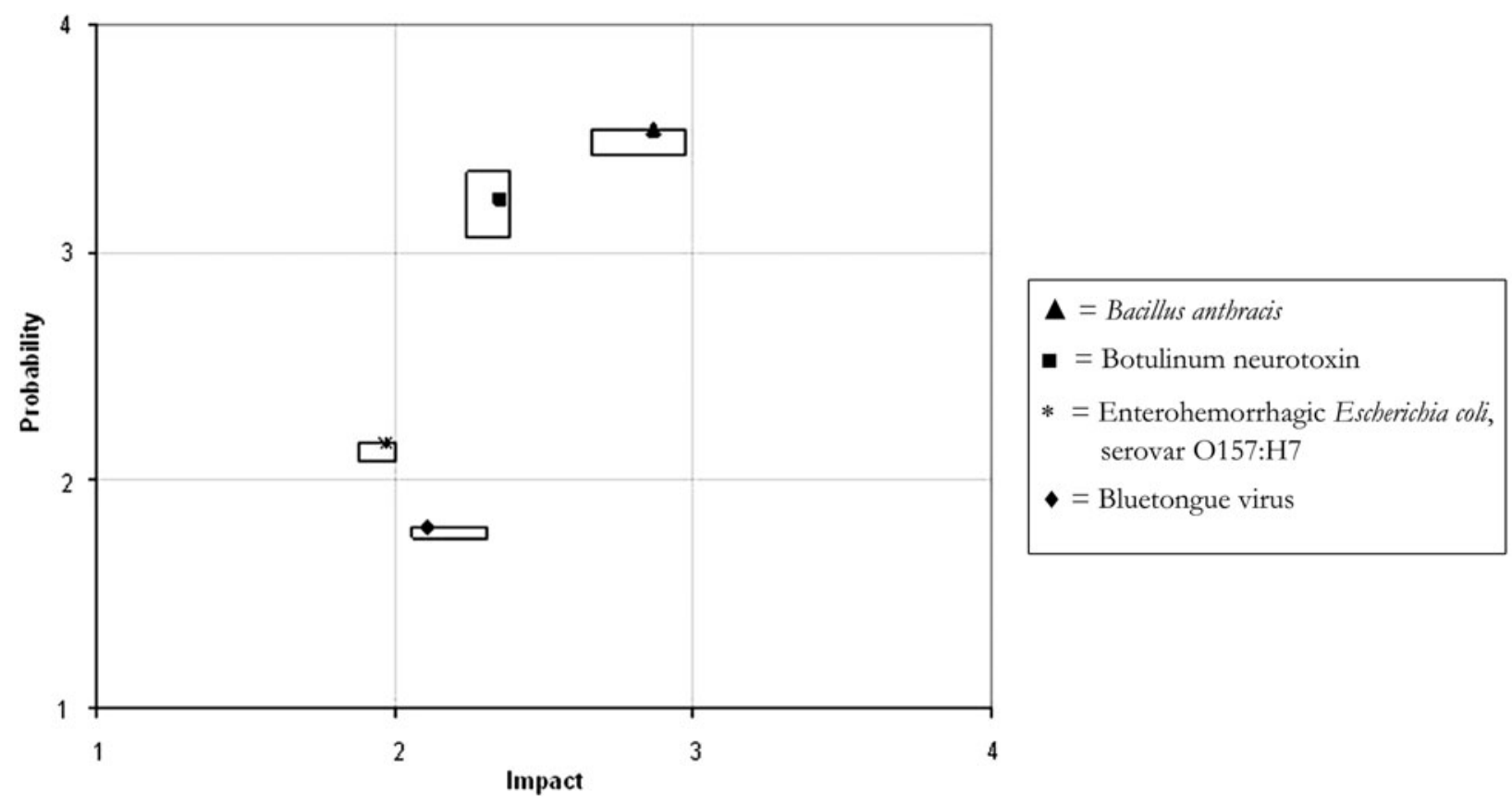

Figure 3. Risk matrix of 4 selected biological agents illustrating probability and impact calculated by the generic risk ranking system. The symbols represent the mean value calculated for the respective agents, whereas the lines of the surrounding box represent possible minimum and maximum values for probability and impact resulting from the data's natural variability and uncertainty on measurelevel. Both axis scales derive from the gradation of classes from 1 to 4 . The more toward the top right the agents are located in the risk matrix, the higher their risk characterized by probability and impact.

absence of specific data for some measures or agents. Criteria for which enough data are not available cannot be ignored by deleting those criteria or measures because this pretends some kind of certainty. There are several possible ways to handle uncertainty caused by a lack of data, each having specific disadvantages: ${ }^{25}$

1. The deletion of measures, as performed by Branquart, ${ }^{26}$ would lead to an incompleteness of information and to misinterpretation for some exotic or newly emerging diseases. The results of the risk ranking would overestimate the relevance of available information. Furthermore, comparative considerations between the agents would not be possible because of a consequently different composition of criteria and foci in the characterization of the pathogens.

2. The assignment of the minimum value to measures with missing information; this would cause an underestimation of the specific criteria and the risk thereof.

3. The attribution of the maximum value to measures, as, for example, by McKenzie et al. ${ }^{20}$ unknown diseases that are mostly insufficient characterized would be overestimated concerning their specific risk.

In order to solve this problem, the measure values were assigned to values ranging from the minimum to the maximum possible item (eg, a case-fatality rate from $0 \%$ to $100 \%)$. Converted into the 4-classes gradation, a minimum class of 1 and a maximum class of 4 with a mean value of 2.5 would arise. As a consequence, a cautious but not overcautious estimation of unknown values was adopted in the calculations through this procedure, a method that has already been used in other publications. ${ }^{27}$

Consequently, the authors decided to mention if there is a lack of data when collecting information on specific highly pathogenic biological agents and to represent the uncertainty through uncertainty boxes. If the available literature is almost complete for the characterization of the agent and natural variation of data is low, the uncertainty boxes are smaller in comparison to a newly emerging disease with lack of data. The less data available for a specific biological agent, the more uncertain becomes the consideration, characterized by larger uncertainty boxes.

Four selected biological agents are presented as an example in a risk-matrix in Figure 3, illustrating their risk, composed of probability and impact. The presented generic risk ranking system poses the unprecedented possibility of categorizing agents with multiple impacts by considering the comprehensive aspects on various populations as described above. Additionally, it includes a system that allows for a weighting to focus on a selected population according to the user's demands. Current systems rank biological agents for specific purposes, such as animal diseases or human public health issues, but do not include criteria for an overall risk ranking. The agents were chosen to exemplify differences and difficulties in illustrating solely animal 
pathogenic agents (bluetongue virus), mainly human pathogenic agents (Enterohemorrhagic Escherichia coli [EHEC] O157:H7), the zoonotic noncontagious Botulinum neurotoxin, and the zoonotic agent Bacillus anthracis. The risk of all these different agents can be calculated likewise by the presented risk ranking system, which allows a direct comparison of the risk posed by human, animal, and zoonotic pathogens. As the developed risk ranking system considers effects on both human and animal populations, those agents that cause damage in both populations are ranked highest, whereas agents that are pathogenic in only 1 population get lower probability and impact scores, since some measures considering the unaffected population are scored with low class affiliation. The uncertainty boxes of different sizes for the 4 selected agents show that the data coverage with regard to information needed for the risk ranking system is more profound for bluetongue virus and EHEC O157:H7 than for Bacillus anthracis and Botulinum neurotoxin (Figure 3).

\section{Discussion}

A new generic risk ranking system for comparing highly pathogenic agents through the consideration of various aspects of the risk related to their potential usage as bioterrorism agents has been developed and was tested for 4 biological agents. The ranking results are based on scientific facts and data (referred to as measures) as they are currently available for each agent. In the presented basic configuration of the ranking system, each measure contributes equally to the score of the corresponding criterion, meaning that it does not matter how many single measures characterize a criterion since all criteria have identical weights against each other. This kind of equal contribution was used in the whole risk ranking system when calculating the risk considering human, animal, and zoonotic aspects in similar amounts. Nevertheless, the weighting of different criteria can be conducted by the user, for example, depending on a unique target population or a specific perception by assigning different weights to categories, criteria, or even measures. This allows the user of the risk ranking system to exclude criteria and categories (by assigning a weight of 0 ) that do not contribute to the risk that a particular biological agent poses to a specific target population. For example, all criteria dealing with human aspects (eg, treatment in human population) are irrelevant when calculating the specific risk for an animal population and could therefore be assigned a weight of 0 .

On the level of measures as describing instrument for the criteria, it is not critical that the measures of different criteria have varying weights in comparison to each other (eg, one-eighth weight of a measure of a criterion described by 8 measures as opposed to a one-quarter weight of a measure of a criterion described by 4 measures), since the measures are used only for the calculation of a data-based score for each criterion. So the score of a criterion and not of a measure determines the risk estimate.

The use of mean values in the calculation led to the approximation of values of more and less hazardous agents at the middle of possible values. ${ }^{25} \mathrm{We}$ are aware of this fact and accept this condition given by the calculation, since the multiplication within the measure, criterion, or category level would lead to an overemphasis of the agents' differences. The authors had to decide whether to obtain the approximation or the spreading out of results and chose the approximation since the pure totaling or multiplication would lead to preweighting due to the different number of measures per criterion.

As soon as specific data for the individual agents have been entered into the database, the criteria set can be used for arbitrarily chosen biological agents.

Comparing results from our risk ranking system with other schema, it becomes evident that there are clear differences, especially for diseases that affect solely the human or the animal population versus agents that can infect both populations. At this juncture, all measures and criteria are weighted equally regardless of whether humans or animals are seen as the target population. However, since a zoonotic agent affects both populations, more measures will be scored higher, resulting in higher mean values for the categories (eg, in the category "accessibility" the measures "number of human cases" and "number of animal cases" are included). Higher values for the criteria lead to higher values for the corresponding categories and consequently to a higher ranked probability and impact that determine the specific risk.

Nevertheless, it is possible to compare agents that are only pathogenic to humans regarding their risk to the human target population through the adjustment of the multiplying weighting factors. The same procedure can be conducted for animal pathogens and their probability and impact in the animal target population. The adaptation of the risk ranking scheme through weighting on measure and criteria levels as well as on the category level dependent on the target population or the considered focus is easy to perform.

In comparison to other risk ranking systems, the measures and criteria of the established system cover almost every subject area and risk connected with a natural, accidental, or deliberate release of highly pathogenic biological agents.

Most other risk ranking systems focus on particular risks, like the introduction into a specific country or the impact solely on the human population. ${ }^{5,14}$ By contrast, the present risk ranking system allows the ranking of high-risk biological agents (human pathogenic, animal pathogenic, and zoonotic agents) with potential for bioterrorism application and can be applied to almost every biological agent. Furthermore, 51 measures describing 28 criteria allow a more comprehensive description of risk by consideration of numerous aspects, compared to systems outlining 
only a smaller number of criteria. ${ }^{5,13,18,28-30}$ Other risk ranking systems have chosen to tackle the complex topic of ranking highly pathogenic biological agents by different methods - for example, a probabilistic decision tree approach. $^{31,32}$

The present system is most suitable for ranking highly pathogenic agents since pathogens that can be found naturally in human and animal populations in high prevalence and that cause simultaneously no symptoms or only mild disease might be ranged higher in probability due to elevated scores (eg, in the accessibility category). Nevertheless, endemic nonhazardous agents would not obtain high total risk scores because of very low results in impact and the multiplying calculation method of risk.

The advantages of the developed risk ranking system for classifying highly pathogenic biological agents may be summarized as follows:

- It is adaptable to different target populations.

- It considers a remarkably broad set of aspects in its criteria.

- It is applicable for low and high data availability, while uncertainty and even natural variation of data are considered and visualized.

- The weighting is adaptable on every level (measures, criteria, and categories), for example, for adaptation to different target populations.

- The class limits of all measures may be adjusted by the user according to particular requirements.

- The formation of a risk value/risk ranking is transparent.

The developed risk ranking system could support the target user group represented by decision makers and stakeholders by providing the needed information and facts that might be important for an extensive risk assessment in emergency situations. The semiquantitative approach, in contrast to the quantitative approach, has the advantage of requiring less time for the collection and evaluation of data while providing all necessary information. The present risk ranking system is intuitively understood by the target user group and could consequently contribute to strategic decisions and an effective immediate reaction in threat situations of natural or deliberate origin. Furthermore, the developed risk ranking can be used to categorize biological agents in risk groups as executed for diverse risk lists (eg, references 2, 4, 33) or to compare agents that are mostly not listed, like bluetongue virus, with agents quoted in these risk lists. It can therefore be used as decision support for researchers and decision makers when considering the classification of newly emerging pathogens (eg, in CBRN legislation). By means of the introduced risk ranking system with its comprehensive approach ranking, experts and scientists can also be assisted in detecting vulnerable points in combination with vulnerability analysis of facilities.
The authors developed a highly adjustable system for the comparable assessment of highly pathogenic biological agents that could be used for bioterrorism threats. It is especially characterized by its broad coverage of aspects within the considered categories and criteria and by its adaptability to specific target populations or to a population spanning analysis.

\section{Acknowledgments}

This research was supported by and executed in the framework of the EU project AniBioThreat (Grant Agreement: Home/2009/ISEC/AG/191) with financial support from the Prevention of and Fight against Crime Programme of the European Union, European Commission-Directorate General Home Affairs. This publication reflects the views only of the authors, and the European Commission cannot be held responsible for any use that may be made of the information contained therein.

\section{REFERENCES}

1. Radosavljevic V, Belojevic G. A new model of bioterrorism risk assessment. Biosecur Bioterror 2009 Dec;7(4):443-451.

2. Bioterrorism agents/diseases. Centers for Disease Control and Prevention website. Updated 2007. http://emergency.cdc.gov/agent/agentlist-category.asp. Accessed February 25, 2011.

3. European Commission. Interim document: technical guidance on generic preparedness planning for public health emergencies. 2005. http://ec.europa.eu/health/ph_threats/Bioterrorisme/keydo_bio_01_en.pdf. Accessed February 25, 2011.

4. Federal Office of Public Health/Switzerland. [B-Terrorism and Food] (in German). 2004.

5. Havelaar AH, van Rosse F, Bucura C, et al. Prioritizing emerging zoonoses in The Netherlands. PLoS One 2010;5(11):e13965.

6. World Health Organization. Public Health Response to Biological and Chemical Weapons: WHO Guidance. 2d ed. Geneva: WHO; 2004. http://www.who.int/csr/delibepidemics/biochemguide/en/. Accessed July 10, 2013.

7. Fosse J, Seegers H, Magras C. Prioritising the risk of foodborne zoonoses using a quantitative approach: application to foodborne bacterial hazards in pork and beef. Rev Sci Tech 2008 Dec;27(3):643-655.

8. Davis RG. Agroterrorism: agents against animals. In: Scanes CG, Miranowski JA, eds. Perspectives in World Food and Agriculture. Iowa City: Iowa State Press; 2004:353-416.

9. Franz DR, Jahrling PB, Friedlander AM, et al. Clinical recognition and management of patients exposed to biological warfare agents. JAMA 1997;278(5):399-411.

10. Irlenkäuser JC. Agroterrorism using the example of animal epizootic diseases. Österreichische Militärische Zeitschrift 2007;2.

11. List of biological agents for export control. Australia Group website. Updated March 2013. http://www.australiagroup. net/en/biological_agents.html. Accessed July 10, 2013. 
12. Australia Group. List of animal pathogens for export control. Australia Group website. Updated June 2011. http://www. australiagroup.net/en/animal.html. Accessed February 25, 2011.

13. Cardoen S, Van Huffel X, Berkvens D, et al. Evidence-based semiquantitative methodology for prioritization of foodborne zoonoses. Foodborne Pathog Dis 2009;6(9):1083-1096.

14. Krause G. How can infectious diseases be prioritized in public health? A standardized prioritization scheme for discussion. EMBO Rep 2008 Jul;9 Suppl 1:S22-S27.

15. World Health Organization. Setting Priorities in Communicable Disease Surveillance. Geneva: WHO; 2006. http://www. who.int/csr/resources/publications/surveillance/WHO_CDS_ EPR_LYO_2006_3/en/index.html. Accessed July 10, 2013.

16. Rotz LD, Khan AS, Lillibridge SR, Ostroff SM, Hughes JM. Public health assessment of potential biological terrorism agents. Emerg Infect Dis 2002 Feb;8(2):225-230.

17. Tegnell A, Van LF, Baka A, et al. Development of a matrix to evaluate the threat of biological agents used for bioterrorism. Cell Mol Life Sci 2006 Oct;63(19-20):2223-2228.

18. Department for Environment Food and Rural Affairs (DEFRA/UK). Documentation for prototype AHW prioritisation decision support tool. December 2006. http://archive.defra.gov. uk/foodfarm/farmanimal/diseases/vetsurveillance/documents/ dst_summary.pdf. Accessed February 25, 2011.

19. Menrath A, Tomuzia K, Frentzel H, Braeunig J, Appel B. Survey of systems for comparative ranking of agents that pose a bioterroristic threat. Zoonoses Public Health. doi: 10.1111/zph.12065.

20. McKenzie J, Simpson H, Langstaff I. Development of methodology to prioritise wildlife pathogens for surveillance. Prev Vet Med 2007;81(1-3):194-210.

21. International Standards Organization. ISO/IEC Guide 73:2009. Risk management - Vocabulary. http://www.iso. org/iso/iso_catalogue/catalogue_tc/catalogue_detail.htm? csnumber $=44651$. Accessed July 10, 2013.

22. Salerno RM, Barnett N, Koelm JG. Balancing security and research at biomedical and bioscience laboratories. SAND No. 2003.0701C. Paper prpesented at: BTR 2003: Unified Science and Technology for Reducing Biological Threats and Countering Terrorism; March 19-21, 2003; Albuquerque, NM. http://cmc.sandia.gov/cmc-papers/sand2003-0701c.pdf. Accessed July 10, 2013.

23. Tucker JB. Biosecurity: Limiting Terrorist Access to Deadly Pathogens. Washington, DC: United States Institute of Peace; 2003. http://www.usip.org/sites/default/files/resources/pwks52.pdf. Accessed July 10, 2013.

24. DISCONTOOLS. Prioritising research to control animal diseases more effectively. The DISCONTOOLS initiative [brochure]. 2011. http://www.discontools.eu/upl/1/default/ doc/1396_DISCONTOOLSbrochurefinal.pdf. Accessed July 10, 2013.

25. Heikkila J. A review of risk prioritisation schemes of pathogens, pests and weeds: principles and practices. Agric Food Sci 2011;20(1):15-28.

26. Branquart E. Guidelines for environmental impact assessment and list classification of non-native organisms in Belgium. ISEIA Guidelines, Harmonia information system 2007;1-4.

27. Petersen K, James W, Thaler A, Ragland R, Hogue A. Use of a priority rating process to sort meatborne zoonotic agents in beef. J Agromed 1996;3(1):17-36.

28. Balabanova Y, Gilsdorf A, Buda S, et al. Communicable diseases prioritized for surveillance and epidemiological research: results of a standardized prioritization procedure in Germany, 2011. PLoS One 2011;6(10).

29. MacIntyre CR, Seccull A, Lane JM, Plant A. Development of a risk-priority score for category A bioterrorism agents as an aid for public health policy. Mil Med 2006 Jul;171(7): 589-594.

30. Pappas G, Panagopoulou P, Akritidis N. Reclassifying bioterrorism risk: are we preparing for the proper pathogens? J Infect Public Health 2009;2(2):55-61.

31. Committee on Methodological Improvements to the Department of Homeland Security's Biological Agent Risk Analysis, National Research Council. Department of Homeland Security Bioterrorism Risk Assessment: A Call for Change. Washington, DC: National Academies Press; 2008. http:// www.nap.edu/catalog/12206.html. Accessed July 10, 2013.

32. Ezell BC, Bennett SP, von Winterfeldt D, Sokolowski J, Collins AJ. Probabilistic risk analysis and terrorism risk. Risk Anal 2010;30(4):575-589.

33. Federal Ministry of Food Agriculture and Consumer Protection/Germany. [Contribution for early detection of bioterroristic attacks on the food chain-A handbook] (in German). 2008.

Manuscript received December 14, 2012;

accepted for publication June 3, 2013.

Address correspondence to: Dr. Katharina Tomuzia Federal Institute for Risk Assessment Department Biological Safety Max-Dohrn-Str. 8-10 10589 Berlin, Germany

E-mail: katharina.tomuzia@bfr.bund.de 